\title{
The Effect of Lightly Gripping a Cane on the Dynamic Balance Control
}

\author{
Kazushige Oshita ${ }^{1, *}$ and Sumio Yano ${ }^{2}$
}

\author{
${ }^{1}$ Department of Sports Science, Faculty of Sports Science, Kyushu Kyoritsu University, 1-8 Jiyugaoka, Yahatanishi-ku, \\ Kitakyushu-city, Fukuoka, 807-8585, Japan; ${ }^{2}$ Graduate School of Human Development and Environment, Kobe Univer- \\ sity, 3-11, Tsurukabuto, Nada-ku, Kobe-city, Hyogo, 657-8501, Japan
}

\begin{abstract}
The purpose of the current study was to investigate the effect of lightly gripping a cane on the Functional

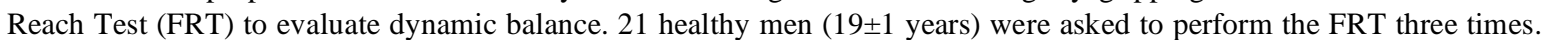
The standard FRT was performed in the first and third trials. In the second trial, participants in a light-grip group $(n=11)$ were told to lightly grip (but to not apply force for mechanical support) the cane during the FRT. Participants in a dependon-cane group $(\mathrm{n}=10)$ were told to perform the FRT while supporting their weight with the cane. FRT is improved by not only supporting a person's own weight with a cane but also just lightly gripping the cane. These findings would be helpful in the development of a useful application to improve the human movement using a haptic sensory supplementation for activities of daily living.
\end{abstract}

Keywords: Dynamic balance, functional reach test, haptic sensory information, light touch.

\section{INTRODUCTION}

Independent mobility is an important factor that affects quality of life, and good balance control is required to decrease the incidence of falls during locomotion. Because postural stability during standing or locomotion is believed to play an important role in balance control, many variables for evaluating postural sway have been suggested (e.g. static or dynamic balance control and balance response of perturbation). Further, researchers have made efforts to improve these variables through various procedures. Researchers have found that providing additional tactile sensory input through the hand or finger improves postural stability during quiet standing [1-9]. Jeka and Lackner [2] showed that lightly touching the tip of the index finger on a fixed surface at waist height (contact force levels that are insufficient for providing mechanical body support; <1 N) resulted in decreased postural sway during tandem Romberg stance. These effects have also been observed for light finger contact with an unstable object. Riley et al. [5] reported that a light finger touch to a cloth curtain decreased postural sway. It has also been found that lightly touching a finger to the upper part of the thighs significantly decreases postural sway during quiet stance $[6,9]$. Furthermore, lightly touching a stable surface can improve the quality of postural responses to slips or trips during quiet standing on a platform by triggering a more rapid corrective response to a sudden displacement of the platform [10]. These findings indicate that lightly touching an object during quiet standing primarily provides information about the relative movement of the body segments and helps an individual sense the movements of the trunk, arms, and thighs relative to one another.

\footnotetext{
*Address correspondence to this author at the Department of Sports Science, Faculty of Sports Science, Kyushu Kyoritsu University, 1-8 Jiyugaoka, Yahatanishi-ku, Kitakyushu-city, Fukuoka, 807-8585, Japan; Tel: +81/ 093/ 693/ 3016; Fax: +81/ 093/ 693/ 3432;

E-mail: monofinswim@gmail.com
}

Although the association between postural sway and light touch has previously been investigated, some researchers have applied these effects to various models of a cane (not real cane). Jeka et al. [11] investigated the effects of lightly gripping a cane placed on a stationary surface on postural control in sighted and congenitally blind individuals. They reported that although postural sway was decreased by lightly gripping a cane on a stationary surface, a slanted cane was far more effective in reducing postural sway than a perpendicular cane. Albertsen et al. [12] found that a light grip is effective even when using an unstable stick support during quiet stance in young healthy individuals. They confirmed that the effect of "light grip" on postural stability during quiet stance is independent from the nature of the cane support (fixed or mobile) when sufficiently large sway related contact forces on the fingers are provided. These findings would be helpful in the development of a useful application for activities of daily living.

However, the most falls and fall related injuries occur during walking or dynamic movement tasks [13, 14]. For instance, Hageman et al. [15] reported that although static balance is not decreased in the elderly until remarkable functional declines occur, dynamic balance decreases much earlier. Riemann et al. [16] and Sell [17] suggested a shift away from static balance testing toward dynamic balance testing as it may be more functional and more applicable to healthy, physically active individuals. Dynamic balance is the ability to anticipate changes in balance and coordinate muscle activity to maintain stability [18], and it means maintaining a stable position while the person undertakes a prescribed movement [19]. It can be evaluated using the Functional Reach Test (FRT) [20], which measures the maximal distance an individual can reach forward beyond arm's length while maintaining a fixed base of support in the standing position. It is a widely used dynamic balance test that can be administered to everyone from children [21] to the elderly [22, 23]. 
If lightly gripping a real cane (not a cane model) improves dynamic balance control (evaluated by the FRT), this finding would be more helpful in the development of a useful application for activities of daily living than that of the previously mentioned studies. Thus, the purpose of the current study was to investigate the effect of lightly gripping a real cane on the FRT score to evaluate dynamic balance.

\section{MATERIALS AND METHODS}

\section{Participants}

Data were obtained from 21 healthy men $(19 \pm 1$ years $)$ with no current or previous medical history of neural, muscular, or skeletal disorders. Participants were randomly assigned to a light-grip (LG group; $\mathrm{n}=11$ ) or a depend-oncane (DC group; $\mathrm{n}=10$ ) group. Before initiating the study, all participants were informed of the purpose of the study and informed consent was obtained from each of them. Further, this study was approved by Human Ethics Committee of Graduate School of Human Development and Environment, Kobe University.

\section{Functional Reach Test (FRT)}

The FRT protocol was based on the previous study by Kage et al. [24], which investigated different FRT methods. The study indicated that the FRT distance and the center of pressure were significantly higher for the one-arm FRT than for the two-arm FRT. They concluded that the one-arm reach is more suitable when evaluating dynamic balance for young adults. Therefore, the FRT score was determined by using the one-arm FRT method in the present study.

Each participant was asked to stand with the lateral aspect of the right arm parallel to the wall. A yardstick was attached to the wall at approximately shoulder height. Participants were then instructed to place their feet a shoulder width apart and flex their dominant arm until it was parallel to the floor while keeping their non-dominant arm at their side. The dominant arm was indicated by each participant based on the hand used to write and throw a ball. This was the right arm for all participants. At this time, the examiner made an initial mark at the participant's middle finger location on the yardstick. The participant was instructed to begin a reaching movement on cue from the examiner and extend the right arm reaching as far forward as possible at normal speed and return to an upright position without any steps while keeping the arms level with the acromion. The FRT score was determined by the displacement of the participant's finger between the initial position and farthest reach attained.

\section{Protocol (Fig. (1))}

Before the experiment, a practice session took place to allow participants to become familiar with the FRT protocol for approximately 5 minutes.

Each participant was asked to perform the FRT three times. The standard FRT was performed in the first and third trials by all participants. In the second trial, participants in the LG group were told to let their left hand lightly grip the cane (just lightly touching of the cane on their fingers, as shown in Fig. (1). Vertical force was not applied). Located to the left anterior oblique position during the FRT. In the second trial, participants in the DC group were told to perform the standard FRT while supporting their weight with the cane held in their left hand. The length of the cane was adjusted so that the top of the cane would reach the crease on the underside of the wrist while standing up straight, arms at the sides. Additionally, the cane length was confirmed when each participant held the cane while standing and flexed the elbow 15 to 20 degrees. During the experiments, the participants were carefully observed by the instructors to ensure compliance with the instruction.

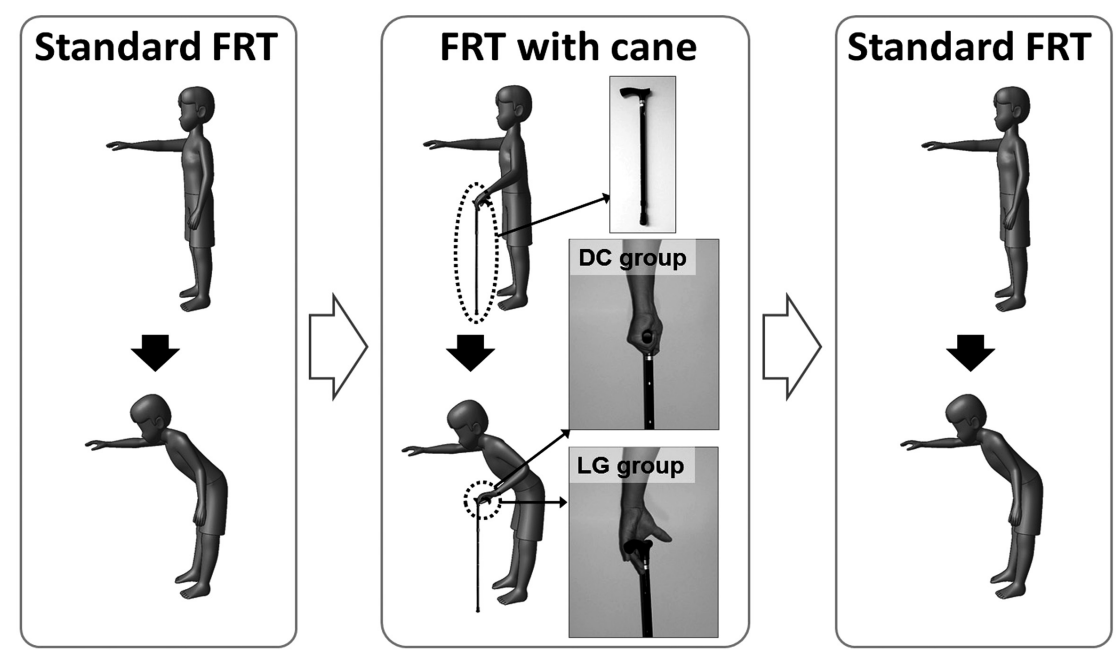

Fig. (1). Schematic diagram of the experimental protocol. Participants were randomly assigned to a light-grip (LG group; $\mathrm{n}=11)$ or a depend-on-cane (DC group; $\mathrm{n}=10$ ) group. The FRT score was determined by using the one-arm (right arm) FRT method in the present study. This FRT (standard FRT) was performed in the first and third trials. In the second trial, participants in the LG group were told to let their left hand lightly grip the cane (not apply vertical force, just lightly touching of the cane on their fingers) located to the left anterior oblique position during the FRT. Participants in the DC group were told to perform the standard FRT while supporting their weight with the cane held in their left hand. 


\section{Statistical Analysis}

Statistical analysis was performed using a one-way repeated-measures analysis of variance (ANOVA), and significant differences between the three different conditions of the FRT in each group were evaluated with post-hoc multiple comparisons using Tukey's test. Values of $p<0.05$ were considered statistically significant. These analyses were performed using J-STAT (ver. 12.5) software. In addition to the significance testing, effect sizes were calculated for the FRT condition effects, using Cohen's $d$.

\section{RESULTS}

The DC group performed three FRT tasks: standard FRT was performed in the first and third trials, and they performed the FRT while supporting their weight with the cane in the second trials. The results are presented in Table $\mathbf{1}$. Although the FRT was not significantly different between standard FRT trials, it was significantly greater in the cane supported trial compared with the two standard trials. These results were constant when the FRT $(\mathrm{cm})$ was normalized to the participant's height $(\mathrm{cm})$. Therefore, dynamic balance is improved when supporting a person's weight with a cane.

The LG group also performed three FRT tasks: standard FRT was performed in the first and third trials, and they performed the FRT while lightly gripping a cane with their fingers and palm (but to not apply force for mechanical support) in the second trials. The results are presented in Table 2. Although the FRT score was not significantly different between the standard FRT trials, it was significantly greater in the light grip trial than in the two standard trials. These results were constant when the FRT $(\mathrm{cm})$ was normalized to the participant's height $(\mathrm{cm})$. Effect size (Cohen's $d$ ) are presented in Table 3. It did present a small sized effect between the standard FRT trials in the both groups. However, it did present a large sized effect in the cane supported trial compared with the two standard trials in the DC group. Further, it did present a medium sized effect in the lightly gripping trial compared with the two standard trials in the LG group. Therefore, dynamic balance is improved by not only supporting a person's own weight with a cane but also just lightly gripping the cane.

\section{DISCUSSION}

This study investigated the effect of lightly gripping a cane on dynamic balance using the FRT. The FRT score was significantly greater when participants supported their weight on a cane and just lightly gripped the cane. Therefore, dynamic balance is significantly improved by not only supporting a person's own weight with a cane but also just lightly gripping the cane.

Contact of the finger or hand with an object improves postural stability during quiet stance. Time of postural stabilization was measured after subjects made light finger contact with a fixed external object by Rabin et al. [7]. They observed that fingertip contact forces stabilized with a time constant of less than $0.5 \mathrm{~s}$ and sway amplitude of the centre of pressure stabilization occurred rapidly following fingertip contact. Further, the stereotypical pattern of force changes at the fingertip correlated with changes in the center of pressure by approximately $300 \mathrm{~ms}$, and was evident within the first $0.5 \mathrm{~s}$ of finger contact. On the other hand, Kouzaki and Masani [25] found that the effects of a light touch during quiet stance were diminished due to loss of finger tactile feedback induced by tourniquet ischemia. Therefore, a light touch during quiet standing provides information about the movement of the body segments by helping an individual sense the movements of the trunk, arms, and thighs relative to one

Table 1. Effect of cane-support on functional reach test (DC-group).

\begin{tabular}{|c|c|c|c|c|}
\hline Variables & $\begin{array}{c}\text { 1. Standard } \\
\text { Mean } \pm \text { S.E.M. }\end{array}$ & $\begin{array}{c}\text { 2. With a Cane } \\
\text { Mean } \pm \text { S.E.M. }\end{array}$ & $\begin{array}{c}\text { 3. Standard } \\
\text { Mean } \pm \text { S.E.M. }\end{array}$ & $\begin{array}{c}\text { ANOVA } \\
\text { F-value, Probability }\end{array}$ \\
\hline \hline Functional Reach (cm) & $46.60 \pm 0.83$ & $62.60 \pm 2.35^{* *}$ & $44.90 \pm 1.26^{\# \#}$ & $F=44.04, P<0.01$ \\
\hline Functional Reach / Height & $0.27 \pm 0.01$ & $0.37 \pm 0.02^{* * *}$ & $0.27 \pm 0.01^{\# \#}$ & $F=30.75, P<0.01$ \\
\hline
\end{tabular}

Each participant was asked to perform the functional reach test (FRT) three times. The standard FRT was performed in the first and third trials. In the second trial, participants performed the FRT while supporting their weight with the cane held in their left hand. Statistical analysis was performed using a one-way repeated-measures analysis of variance (ANOVA), and significant differences between the three different conditions of the FRT in each group were evaluated with post-hoc multiple comparisons using Tukey's test.

**; $<<0.01$ vs. "1 Standard" (Tukey's test)

$\# \# ; \mathrm{p}<0.01$ vs. "2 with a cane" (Tukey's test).

Table 2. Effect of lightly grip to cane on functional reach test (LG-group).

\begin{tabular}{|c|c|c|c|c|}
\hline Variables & $\begin{array}{c}\text { 1. Standard } \\
\text { Mean } \pm \text { S.E.M. }\end{array}$ & $\begin{array}{c}\text { 2. With a Cane } \\
\text { Mean } \pm \text { S.E.M. }\end{array}$ & $\begin{array}{c}\text { 3. Standard } \\
\text { Mean } \pm \text { S.E.M. }\end{array}$ & $\begin{array}{c}\text { ANOVA } \\
\text { F-value, Probability }\end{array}$ \\
\hline \hline Functional Reach (cm) & $45.00 \pm 1.39$ & $47.91 \pm 1.15^{*}$ & $44.36 \pm 1.59^{\#}$ & $F=6.20, P=0.01$ \\
\hline Functional Reach / Height & $0.26 \pm 0.01$ & $0.28 \pm 0.01^{*}$ & $0.26 \pm 0.01^{\#}$ & $F=6.17, P=0.01$ \\
\hline
\end{tabular}

Each participant was asked to perform the functional reach test (FRT) three times. The standard FRT was performed in the first and third trials. In the second trial, participants were told to let their left hand lightly grip the cane (not apply vertical force, just lightly touching of the cane on their fingers (Fig. 1)). Statistical analysis was performed using a one-way repeated-measures analysis of variance (ANOVA), and significant differences between the three different conditions of the FRT in each group were evaluated with post-hoc multiple comparisons using Tukey's test.

*; p 0.05 vs. "1 Standard" (Tukey's test)

$\# ; \mathrm{p}<0.05$ vs. "2 with a cane" (Tukey's test). 
Table 3. Effect size (Cohen's $d$ ) about the effect of cane on functional reach test.

\begin{tabular}{|c|c|c|c|}
\hline \multirow[b]{2}{*}{ Cohen's $d$} & \multicolumn{3}{|c|}{ Functional Reach Test } \\
\hline & $\begin{array}{l}\text { 1. Standard } v s \text {. } \\
\text { 2. With a Cane }\end{array}$ & $\begin{array}{l}\text { 3. Standard vs. } \\
\text { 2. With a Cane }\end{array}$ & $\begin{array}{l}\text { 1. Standard vs. } \\
\text { 3. With a Cane }\end{array}$ \\
\hline LG (light-grip) group & 0.69 & 0.77 & 0.13 \\
\hline DC (depend-on-cane) group & 2.64 & 2.95 & 0.41 \\
\hline \multirow[b]{2}{*}{ Cohen's $d$} & \multicolumn{3}{|c|}{ Functional Reach Test / Height } \\
\hline & $\begin{array}{l}\text { 1. Standard } v s \text {. } \\
\text { 2. With a Cane }\end{array}$ & $\begin{array}{l}\text { 3. Standard } v s \text {. } \\
\text { 2. With a Cane }\end{array}$ & $\begin{array}{l}\text { 1. Standard vs. } \\
\text { 3. With a Cane }\end{array}$ \\
\hline LG (light-grip) group & 0.59 & 0.72 & 0.12 \\
\hline
\end{tabular}

another. The present study investigated whether lightly gripping a real cane improves dynamic balance. The results show that dynamic balance evaluated by the FRT was significantly improved by lightly gripping a cane. Therefore, the present results indicate that the effects of lightly gripping a cane on postural stability can be applied to dynamic postural stability.

Although the present study revealed the effects of lightly gripping a cane on dynamic balance, the mechanisms underlying the association between postural sway and light touch is yet to be thoroughly examined. When an individual maintains a quiet stance, plantar flexors are recruited as antigravity muscles at the ankle joint. Plantar flexor activity during quiet stance differs under various conditions. It is greater in the elderly than in young adults [26-28], with eyes closed than with eyes open [29], and on an unstable surface than on a stable surface [30]. However, some researcher reported that plantar flexor (soleus) activity was not significantly changed by a light touch during quiet stance $[8,9]$.

Although dorsiflexor activity during quiet stance usually remains low (i.e. plantar flexors are agonist muscles and dorsiflexors are antagonist muscles), it influences postural stability. During the bipedal stance, muscle co-activation of the ankle joint increased with age [26, 27], and persons with less physical function during postural control tasks showed higher muscle co-activation of the ankle joints [27, 31]. Further, Oshita and Yano [9] reported that although soleus (plantar flexor) activity did not significantly change with a light touch during quiet stance, tibialis anterior (dorsiflexor) activity significantly decreased. Therefore, a light touch during quiet stance might improve postural stability by decreasing co-activation of the ankle muscles, and provide information about the movement of the body segments. Some researchers have found unfavourable effects of co-activation on the motion of postural control, reporting that excessive muscular co-activation increases the rigidity of postural control $[32,33]$. Rigidity of postural control induced by greater muscle co-activation reduces the degrees of freedom during postural control [34]. The FRT requires good postural control as participants have to maintain their posture. Therefore, if co-activation is decreased by light touch, the FRT score will improve. Actually, Nagai et al. [31] found that balance training improves the FRT score and that other dynamic balance tests were associated with decreased muscle coactivation in the ankle joint. Therefore, the effects of lightly gripping a cane on the FRT score might be induced by the decreasing co-activation of ankle muscles.

The association between postural stability and light touch is scientifically interesting. However, it cannot be applied to various movements (i.e., daily activities) during contact with a fixed stable object. The present study revealed that dynamic balance was improved not only by supporting a person's weight on a real cane but also by just lightly gripping the cane. A cane can be used while performing various movements. Therefore, our results suggest a potential new use for a cane. However, if individuals want to maintain their posture, they can support their body weights by strongly holding on to objects (i.e., a wall, handrail, or cane). This can help them stand independently while greatly reducing their risk of falling with progressive leg weakness. However, this may cause weakening of muscle function in persons who have enough muscle strength to stabilize their own postures because using a cane greatly reduces muscular force outputs needed for posture stability [35]. By contrast, lightly touching a cane does not reduce muscular force outputs while maintaining posture because contact force levels are insufficient for providing mechanical body support. Furthermore, some studies revealed that the association between postural control and haptic input through light touching affects motor learning $[9,36]$. These reports suggest that light touching is a potential training tool to acquire postural control ability. If the effect of lightly gripping a cane on postural stability is also relevant during various motions, lightly touching a cane might be useful in improving human movement. Therefore, further studies are needed to clarify the direct relationship between muscle activity or joint kinematics and the effect of light touch.

\section{CONFLICT OF INTEREST}

The authors confirm that this article content has no conflict of interest. 


\section{ACKNOWLEDGEMENTS}

This work was supported by Grant-in-Aid for Young Scientists (B) (25870988), and Grant-in-Aid for Scientific Research (C) (26350659).

\section{PATIENT'S CONSENT}

Before initiating the study, all participants were informed of the purpose of the study and informed consent was obtained from each of them.

\section{REFERENCES}

[1] M. Holden, J. Ventura, and J.R. Lackner, "Stabilization of posture by precision contact of the index finger", J. Vestib. Res., vol. 4, pp. 285-301, 1994.

[2] J.J. Jeka, and J.R. Lackner, "Fingertip contact influences human postural control", Exp. Brain Res., vol. 100, pp. 495-502, 1994.

[3] J.J. Jeka, and L.R. Lackner, "The role of haptic cues from rough and slippery surfaces in human postural control", Exp. Brain Res., vol. 103, pp. 267-276, 1995.

[4] S. Clapp, and A.M. Wing, "Light touch contribution to balance in normal bipedal stance", Exp. Brain Res., vol. 125, pp. 521-524, 1995.

[5] M.A. Riley, T.A. Stoffregen, M.J. Grocki, and M.T. Turvey, "Postural stabilization for the control of touching", Hum. Mov. Sci., vol. 18, pp. 795-817, 1999.

[6] A. Nagano, S. Yoshioka, D.C. Hay, and S. Fukashiro, "Light finger touch on the upper legs reduces postural sway during quasi-static standing", Motor Control, vol. 10, pp. 348-358, 2006.

[7] E. Rabin, P. DiZio, and J.R. Lackner, "Time course of haptic stabilization of posture", Exp. Brain Res., vol. 170, pp. 122-126, 2006.

[8] S. Watanabe, K. Kobara, and H. Ishida, "Influence of fingertip contact with a wall on postural sway and electromyographyic activity of the soleus muscle", Electromyogr. Clin. Neurophysiol., vol. 50, pp. 229-233, 2010.

[9] K. Oshita, and S. Yano, "Effects of light finger touch to the upper legs on postural sway and muscle activity during quiet standing", Conf. Proc. IEEE Eng. Med. Biol. Soc., vol. 2013, pp. 7459-7562, 2013.

[10] R. Dickstein, R.J. Peterka, and F.B. Horak, "Effects of light fingertip touch on postural responses in subjects with diabetic neuropathy", J. Neurol. Neurosurg. Psychiatry., vol. 74, pp. 620-626, 2003.

[11] J. Jeka, R.D. Easton, B.L. Bentzen, and J.R. Lackner, "Haptic cues for postural control in sighted and blind individuals", Percept. Psychophys., vol. 58, pp. 409-423, 1996.

[12] I.M. Albertsen, J.J. Temprado, and E. Berton, "Effect of haptic supplementation on postural stabilization: A comparison of fixed and mobile support conditions", Hum. Mov. Sci., vol. 29, pp. 9991010, 2010.

[13] A.J. Campbell, M.J. Borrie, and G.F. Spears, "Risk factors for falls in a community-based prospective study of people 70 years and older”, J. Gerontol., vol. 44, pp. M112-M1117, 1989.

[14] K.P. Granata, and T.E. Lockhart, "Dynamic stability differences in fall-prone and healthy adults", J. Electromyogr. Kinesiol., vol. 18, pp. 172-178, 2008.

[15] P.A. Hageman, J.M. Leibowitz, and D. Blanke, "Age and gender effects on postural control measures", Arch. Phys. Med. Rehabil., vol. 76, pp. 961-965, 1995.

[16] B.L. Riemann, N.A. Caggiano, and S.M. Lephart, "Examination of a clinical method of assessing postural control during a functional performance task", J. Sport Rehabil., vol. 8, pp. 171-183, 1999.
[17] T.C. Sell, "An examination, correlation, and comparison of static and dynamic measures of postural stability in healthy, physically active adults", Phys. Ther. Sport, vol. 13, pp. 80-86, 2011.

[18] M.E. Rogers, N.L. Rogers, N. Takeshima, and M.M. Islam, "Methods to assess and improve the physical parameters associated with fall risk in older adults", Prev. Med., vol. 36, pp. 255-264, 2003.

[19] M.T. Karimi, and S. Solomonidis, "The relationship between parameters of static and dynamic stability tests" J. Res. Med. Sci., vol. 16, pp. 530-535, 2011.

[20] P.W. Duncan, D.K. Weiner, J. Chandler, and S. Studenski, "Functional reach: a new clinical measure of balance", J. Gerontol., vol 45, pp. M192-M197, 1990.

[21] R.A. Norris, E. Wilder, and J. Norton, "The functional reach test in 3- to 5-year-old children without disabilities", Pediatr. Phys. Ther., vol. 20, pp. 47-52, 2008.

[22] P. Duncan, S. Studenski, J. Chandler, and B. Prescott, "Functional Reach: Predictive validity in a sample of elderly male veterans", $J$. Gerontol., vol. 47, pp. M93-M98, 1992.

[23] J. Davis, P. Ross, M. Nevitt, and R. Wasnich, "Risk factors for falls and for serious injuries on falling among older Japanese women in Hawaii”, J. Am. Geriatr. Soc., vol. 47, pp. 792-798, 1999.

[24] H. Kage, M. Okuda, I. Nakamura, I. Kunitsugu, S. Sugiyama, and T. Hobara, "Comparison of the one-arm and two-arm functional reach test in young adults", J. Phys. Ther. Sci., vol. 21, pp. 207212, 2009.

[25] M. Kouzaki, and K. Masani, "Reduced postural sway during quiet standing by light touch is due to finger tactile feedback but not mechanical support", Exp. Brain Res., vol. 188, pp. 153-158, 2008.

[26] I. Melzer, N. Benjuya, and J. Kaplanski, "Age-related changes of postural control: effect of cognitive tasks", Gerontology, vol. 47, pp. 189-194, 2001.

[27] K. Nagai, M. Yamada, K. Uemura, Y. Yamada, N. Ichihashi, and T. Tsuboyama, "Differences in muscle coactivation during postural control between healthy older and young adults", Arch. Gerontol. Geriatr., vol. 53, pp. 338-343, 2011.

[28] S. Baudry, and J. Duchateau, "Age-related influence of vision and proprioception on Ia presynaptic inhibition in soleus muscle during upright stance", J. Physiol., vol. 590, pp. 5541-5554, 2012.

[29] F.L.A. Braun, W.M. Pereira, L.P. Rossi, I.I. Kerpers, A. Jr. Rodrigues de Paula, and C.S. Oliveira, "Analysis of electromyographic activity of ankle muscles on stable and unstable surfaces with eyes open and closed", J. Bodyw. Mov. Ther., vol. 15, pp. 496-501, 2011.

[30] P.G. Morasso, and M. Schieppati, "Can muscle stiffness alone stabilize upright standing?", J. Neurophysiol., vol. 82, pp. 1622$1626,1999$.

[31] K. Nagai, M. Yamada, B. Tanaka, K. Uemura, S. Mori, T. Aoyama, N. Ichihashi, and T. Tsuboyama, "Effects of balance training on muscle coactivation during postural control in older adults: a randomized controlled trial", J. Gerontol. A Biol. Sci. Med. Sci., vol. 67, pp. 882-889, 2012.

[32] P.F. Tang, and M.H. Woollacott, "Inefficient postural responses to unexpected slips during walking in older adults", J. Gerontol. A Biol. Sci. Med. Sci., vol. 53, pp. M471-M480, 1998.

[33] G. Wu, "Age-related differences in Tai Chi gait kinematics and leg muscle electromyography: a pilot study", Arch. Phys. Med. Rehabil., vol. 89, pp. 351-357, 2008.

[34] M.G. Tucker, J.J. Kavanagh, R.S. Barrett, and S. Morrison, “Agerelated differences in postural reaction time and coordination during voluntary sway movements", Hum. Mov. Sci., vol. 27, pp. 728737, 2008.

[35] E.F. Murphy, "Some notes on canes and cane tips", Bull. Prosthet. Res., vol. 10-4, pp. 65-76, 1965.

[36] L. Johannsen, S.Z. Lou, and H. Y. Chen, "Effects and after-effects of voluntary intermittent light finger touch on body sway", Gait Posture, vol. 40, pp. 575-580, 2014.

(c) Oshita and Yano; Licensee Bentham Open.

This is an open access article licensed under the terms of the Creative Commons Attribution Non-Commercial License (http://creativecommons.org/licenses/by-nc/3.0/) which permits unrestricted, non-commercial use, distribution and reproduction in any medium, provided the work is properly cited. 